\title{
Comportamento ingestivo de novilhos em pastagem nativa no Rio Grande do Sul
}

\author{
Cassiano Eduardo Pinto ${ }^{1}$, Paulo César de Faccio Carvalho², Adriana Frizzo ${ }^{3}$, José Acélio \\ Silveira da Fontoura Júnior ${ }^{4}$, Carlos Nabinger ${ }^{2}$, Rubson Rocha ${ }^{5}$
}

\author{
1 M. Sc., EPAGRI. Caixa Postal 181, CEP: 88502-170. \\ 2 Departamento de Plantas Forrageiras e Agrometeorologia, UFRGS. \\ 3 Doutoranda, Departamento de Plantas Forrageiras e Agrometeorologia. \\ ${ }^{4}$ M. Sc. PRODUZ. \\ 5 Med. Vet. Dr., EPAGRI.
}

RESUMO - Neste estudo avaliou-se a influência da variação nas ofertas de fitomassa ao longo das diferentes estações de crescimento na dinâmica da pastagem e no rendimento animal. Os tratamentos consistiram de quatro ofertas fixas de fitomassa $(4,0 ; 8,0 ; 12,0$ e 16,0\%, expresso em kg de MS/100 kg de PV por dia) ao longo da estação de crescimento e três ofertas variáveis ( $8,0 \%$ na primavera e $12,0 \%$ no restante da estação de crescimento; $12,0 \%$ na primavera e $8,0 \%$ no restante da estação de crescimento; e $16,0 \%$ na primavera e $12,0 \%$ no restante da estação de crescimento). O delineamento experimental foi o de blocos completamente casualizados com duas repetições. O método de pastejo foi o contínuo com lotação variável, utilizando-se a técnica de put-and-take. As produções primária e secundária e o comportamento de novilhos de sobreano foram medidos regularmente. Os resultados comprovaram que situações de oferta de forragem muito baixa, como no tratamento 4,0\%, penalizam fortemente o desempenho dos animais. O tratamento de $8,0 \%$ na primavera e de $12,0 \%$ no restante da estação de crescimento promoveu bons ganhos de peso, sugerindo que esta prática pode ser interessante quando se pretende manipular a estrutura da pastagem. Os resultados do comportamento ingestivo dos animais indicaram que, diferentemente do que ocorre em pastagens cultivadas, a oferta de forragem e a massa de forragem não explicam suficientemente o tempo de pastejo dos animais. Em vegetações heterogêneas, deve-se considerar a diversidade estrutural na caracterização da pastagem visando sua associação ao comportamento em pastejo.

Palavras-chave: comportamento em pastejo, oferta de forragem, pastagem nativa

\section{Ingestive behaviour of steers on natural grasslands of Rio Grande do Sul}

\begin{abstract}
This study tested the hypothesis that variation of herbage allowance during the growing season influences pasture dynamics and grazing behaviour. Treatments consisted of four fixed herbage allowances during the entire year (4.0, $8.0,12.0$, and $16.0 \%$, expressed as $\mathrm{kg} \mathrm{DM} / 100 \mathrm{~kg}$ live weight/day) and three variable herbage allowances (8\% in spring and $12 \%$ along the rest of the grazing season; $12 \%$ spring and $8 \%$ along the rest of the grazing season; $16 \%$ in spring and $12 \%$ along the rest of the grazing season). A randomized complete block design was used, with two replicates. It was used a variable continuous stocking method, using the "put-and-take" technique. Primary and secondary productions were measured, as well as steer grazing behaviour. Results showed that low herbage allowance, as in $4 \%$ treatment, jeopardize animal performance. Herbage allowance of $8 \%$ in spring and $12 \%$ along the rest of the grazing season promoted good animal performance, suggesting this management could be interesting when manipulating sward structure. Concerning grazing behaviour, results indicated that differently from what occurs in cultivated pastures, herbage allowance or herbage mass did not explain sufficiently the grazing time observed. It was concluded that in heterogeneous vegetation, it must be considered the structural diversity on pasture characterization in order to link that with grazing behaviour.
\end{abstract}

Key Words: grazing behaviour, herbage allowance, native pasture

\section{Introdução}

O ecossistema pastagem nativa ocupa aproximadamente $44 \%$ da superfície do Rio Grande do Sul e constitui o principal substrato forrageiro para a pecuária gaúcha desse estado. Essa pastagem tem como característica enorme riqueza florística, com aproximadamente 400 espécies de gramíneas e 150 espécies de leguminosas peculiares à altitude, ao clima e ao solo de cada região (Boldrini, 1997). Portanto, estudos sobre o uso sustentável deste importante recurso forrageiro são de extremo valor, tanto do ponto de vista econômico quanto ambiental.

A relação funcional entre oferta de forragem $(\mathrm{OF}) \mathrm{e}$ desempenho animal tem sido investigada em pastagens 
nativas da Depressão Central do Rio Grande do Sul. Segundo Maraschin (1998), nas pressões de pastejo média a leve, com a oportunidade de algum pastejo seletivo, a pastagem cresce mais, produz mais e possibilita $0,5 \mathrm{~kg}$ de ganho médio diário (GMD) por animal. Os níveis de utilização da pastagem determinam, ao longo dos anos, duas estruturas bem definidas: estrato inferior, composto por espécies de hábito de crescimento prostrado, como Paspalum notatum Fl. e Paspalum paucifolium Michx; e estrato superior, formado por gramíneas eretas, comoAndropogon lateralis Nees, Aristida filifolia (Arech.) e Eryngium horridum. Setelich (1994) destacou que, em maiores ofertas de forragem (OF), ocorre formação de touceiras, aumentando a participação de gramíneas de baixo valor forrageiro. $\mathrm{O}$ grau de pastejo das touceiras também varia segundo as espécies e a OF, pois o pastejo das touceiras aumenta à medida que diminui a OF.

As relações envolvendo a OF e o desempenho animal e seus efeitos sobre a estrutura da pastagem e a composição botânica têm sido abordadas na literatura (Escosteguy, 1990; Moojen, 2002; Corrêa, 1993; Setelich, 1994; Maraschin, 1998), entretanto, as estratégias estabelecidas pelos animais pastejando ambientes complexos e heterogêneos, como as pastagens naturais, ainda não foram suficientemente esclarecidas.

A estrutura da pastagem, entendida como distribuição horizontal e vertical da forragem, influencia significativamente o comportamento ingestivo dos animais. Hogdson (1985) discutiu a importância da estrutura em relação à seleção da dieta, assinalando as dificuldades de acesso a todos os estratos do perfil em situações de pastagens fechadas de gramíneas temperadas. Esse autor considera a altura determinante do tamanho do bocado nas pastagens temperadas, enquanto, nas pastagens tropicais, a densidade das pastagens seria mais importante.

A disponibilidade de MS afeta a proporção de forragem que pode ser colhida pelo animal, influenciando seu grau de seletividade e consumo. Segundo Poppi et al. (1987), a massa de forragem oferecida influencia a curva de resposta do consumo do animal, pois altera a estrutura da pastagem por meio de seus componentes altura e/ou densidade. Minson (1983) citou $1.000 \mathrm{~kg}$ de MS/ha comoo valor crítico de disponibilidade em uma pastagem. Ribeiro Filho et al (1997), avaliando o tempo de pastejo de novilhos submetidos a OF crescentes, encontraram decréscimo no tempo de pastejo de $0,43 \mathrm{~h} /$ dia por unidade percentual de aumento na OF.

A maioria dos trabalhos avaliando comportamento ingestivo foi desenvolvida em pastagens temperadas monoespecíficas ou com consorciações entre duas espécies. Há uma grande lacuna a ser preenchida pela experimentação agronômica no tocante ao comportamento ingestivo em ambientes de pastejo heterogêneos como as pastagens naturais.

Este trabalho foi realizado com objetivo de investigar a influência da variação da oferta de forragem ao longo da estação de crescimento na dinâmica e no comportamento ingestivo em pastagem natural no Rio Grande do Sul.

\section{Material e Métodos}

O experimento foi conduzido em uma pastagem natural da Depressão Central do Rio Grande do Sul localizada a $30^{\circ} 05^{\prime} 52^{\prime \prime}$ de latitude sul, $51^{\circ} 39^{\prime} 08^{\prime \prime}$ de longitude oeste e $46 \mathrm{~m}$ de altitude. O clima da região é do tipo $\mathrm{Cfa}$, segundo classificação de Köppen, e o solo é do tipo podzólico vermelho amarelo plíntico nas áreas planas, com áreas alagadiças na parte baixa, formadas por solos hidromórficos tipo gley húmico.

O período experimental totalizou 202 dias de estação de crescimento da pastagem natural (04/10/2001 a 23/04/2002), no qual foram avaliadas sete combinações de oferta de fitomassa seca total (OFMST), arranjadas da seguinte forma:

$4,0=4 \mathrm{~kg}$ de MS $/ 100 \mathrm{~kg}$ de PV/dia durante toda a estação de crescimento;

$8,0=8 \mathrm{~kg}$ de $\mathrm{MS} / 100 \mathrm{~kg}$ de $\mathrm{PV} /$ dia durante toda a estação de crescimento;

$12,0=12 \mathrm{~kg}$ de $\mathrm{MS} / 100 \mathrm{~kg}$ de $\mathrm{PV} /$ dia durante toda a estação de crescimento;

$16,0=16 \mathrm{~kg}$ de $\mathrm{MS} / 100 \mathrm{~kg}$ de $\mathrm{PV} /$ dia durante toda a estação de crescimento;

$8 \mathrm{p} 12=8 \mathrm{~kg}$ de $\mathrm{MS} / 100 \mathrm{~kg}$ de $\mathrm{PV} /$ dia na primavera e $12 \mathrm{~kg}$ de MS/100 kg de PV/dia no restante da estação de crescimento;

$12 \mathrm{p} 8=12 \mathrm{~kg}$ de $\mathrm{MS} / 100 \mathrm{~kg}$ de $\mathrm{PV} /$ dia na primavera e $8 \mathrm{~kg}$ de MS/100 kg de PV/dia no restante da estação de crescimento; e

$16 \mathrm{p} 12=16 \mathrm{~kg}$ de MS/100 kg de PV/dia na primavera e $12 \mathrm{~kg}$ de MS/100 kg de PV/dia no restante da estação de crescimento.

Os tratamentos com OF fixas têm sido conduzidos de forma quase ininterrupta desde 1986 , sempre no período de crescimento da pastagem nativa. Durante o período de 2000 a 2001, Soares (2005) conduziu esta área com os mesmos tratamentos, porém alterando pela primeira vez as ofertas de forragem. Alterou-se a OF nos tratamentos pertinentes quando do aparecimento de pelo menos 30 inflorescências de Paspalum notatum por $\mathrm{m}^{2}$ no tratamento $12,0 \%$. Consi- 
derou-se este o indicativo da transição entre estádios fenológicos e escolheu-se o $P$. notatum como espécie indicadora por ser esta uma das principais espécies componentes da flora local.

Para o cálculo da OFMST, foram consideradas espécies de ambos os estratos inferior e superior. Foram utilizados novilhos de sobreano $(251 \mathrm{~kg} \pm 50)$, três animais experimentais por unidade experimental, escolhidos aleatoriamente dentro do rebanho disponível. O delineamento experimental foi o de blocos completamente casualizados e o método de pastejo foi o contínuo com lotação variável, conforme técnica put-and-take descrita por Mott \& Lucas (1952).

A estimativa da fitomassa foi realizada a cada 28 dias, durante cada ajuste da carga, utilizando-se o método da dupla amostragem (Wilm et al., 1944).

A taxa de acúmulo de fitomassa foi estimada pelo método do triplo emparelhamento (Moraes, 1995), com adaptação da metodologia proposta por Klingman et al. (1943), utilizando-se gaiolas de exclusão ao pastejo. As avaliações foram feitas a cada 28 dias, distribuindo-se aleatoriamente quatro gaiolas por unidade experimental. As amostras de forragem foram cortadas rente ao solo com uma tesoura elétrica de esquila, em uma área circular de $0,25 \mathrm{~m}^{2}$. As amostras foram acondicionadas em sacos de papel e secas em estufa de ar forçado a $65^{\circ} \mathrm{C}$ até atingirem peso constante.

A produção total de fitomassa foi calculada pelo somatório do acúmulo de cada subperíodo. A disponibilidade de fitomassa de cada período foi obtida somando-se a taxa de acúmulo correspondente. As médias de fitomassa disponível, ponderadas pelo número de dias de cada subperíodo, permitiram obter os valores do período total de avaliação.

A oferta de fitomassa observada foi obtida pela razão entre a carga animal média e a disponibilidade de MS do período correspondente multiplicada por 100 .

A caracterização da vegetação foi realizada percorrendo-se as unidades experimentais em transectas transversais imaginárias ao longo de toda a área. A cada dez passos, com auxílio de uma régua graduada (sward-stick), efetuou-se a leitura de altura do dossel, identificando-se o estrato vegetativo e a espécie. Foram feitas aproximadamente 80 leituras por unidade experimental, sendo três caracterizações antecedendo as avaliações de comportamento ingestivo.

O GMD foi calculado por meio da diferença de peso dos animais experimentais no início e ao final do período experimental. Os animais foram pesados após jejum de 16 horas e o ganho por área $(\mathrm{G} / \mathrm{ha}$ ) foi obtido multiplicando-se o GMD por animais-dia/ha de cada período, expresso em $\mathrm{kg}$ de PV/ha.
O comportamento ingestivo foi observado em intervalos de dez minutos, verificando-se visualmente a atividade dos três animais experimentais em cada uma das unidades experimentais. Foram realizadas três avaliações nos dias 30 de janeiro, 05 de março e 10 de abril de 2002, do amanhecer ao pôr-do-sol. Os valores, em minutos, foram registrados como tempo de pastejo (TP), tempo de ruminação (TR) e tempo de descanso (TD). Os dados específicos e correspondentes ao período de avaliação do comportamento ingestivo foram submetidos à análise de variância e à comparação de médias pelo teste Tukey, a 5\% de significância, utilizando-se o pacote estatístico SAS (SAS, 1994), para análise dos efeitos de tratamento, período e interações. As avaliações de comportamento ingestivo foram realizadas em dias típicos de verão, com temperaturas máximas de 33,$4 ; 35,1$ e $30,8^{\circ} \mathrm{C}$ e mínimas de 16,$9 ; 19,6$ e $18^{\circ} \mathrm{C}$ para as avaliações dos dias 30 de janeiro, 05 de março e 10 de abril, respectivamente.

O estudo global do conjunto das 12 variáveis foi realizado por análise multivariada, evidenciando-se as ligações, semelhanças ou diferenças, por meio do pacote estatístico Statistica (STATSOFT, 1998), móduloFactor Analysis. A consideração dos valores dos componentes principais, ou mesmo a análise não correlacionada pode tornar muito mais fácil a compreensão do significado dos dados. Um procedimento de análise fatorial foi iniciado tomando-se alguns componentes principais como fatores. Esses fatores iniciais puderam, então, ser modificados por meio de um processo particular de transformação, denominado rotação fatorial, de modo a torná-los de mais fácil interpretação. Para reduzir o número de variáveis a serem analisadas, utilizaram-se $\mathrm{r}^{1}$ e ${ }^{2}$ como repetição 1 e repetição 2 , respectivamente, com o método fatorial de análise dos componentes principais.

\section{Resultados e Discussão}

Os resultados obtidos em cada unidade experimental, referentes a cada variável analisada, são apresentados na Tabela 1.

Foram contempladas, no fator 1 , as variáveis oferta de forragem real (OF_REAL), freqüência do estrato superior (FREQ_SU) e massa de forragem (MAS_FOR); no fator 2, a freqüência do estrato inferior (FREQ_INF); no fator 3, a oposição entre tempo de pastejo (TP) e altura do estrato inferior (ALT_INF); e, no fator 4, a altura do estrato superior (ALT_SUP). Em vez de utilizar toda as informações contidas nas 12 variáveis originais, com a utilização da combinação linear de apenas quatro variáveis, é possível representar mais que $72 \%$ da variância total dos dados. 
Pinto et al.

Tabela 1 - Média dos dados referentes aos períodos de avaliação do comportamento ingestivo Table 1 - Average across evaluation periods of ingestive behaviours

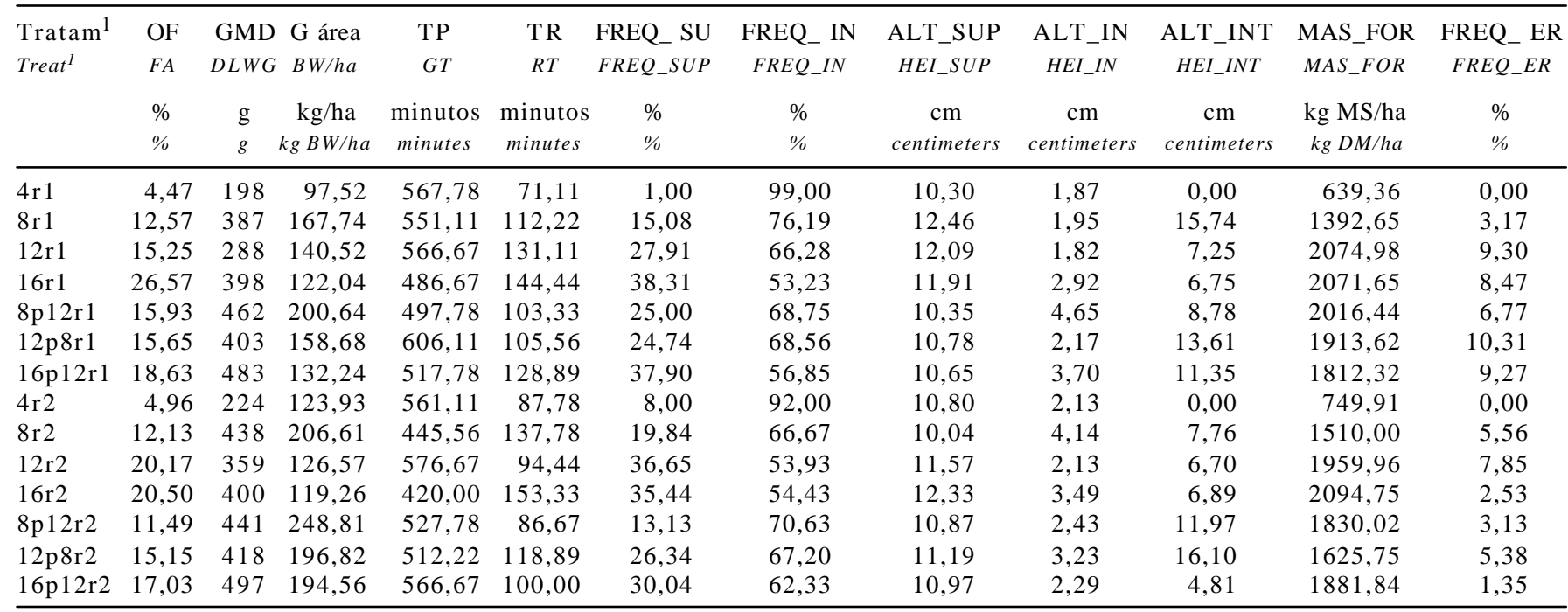

1 Tratamentos (Treatments), Oferta de forragem - OF (Forrage allowance - FA), Ganho por área - G área (Body weight per ha), Tempo de pastejo - TP (Grazing time - GT), Tempo de ruminação (Rumination time - RT), Freqüência do estrato superior - FREQ SU (Frequency superior strata -FREQ SUP), Freqüência do estrato inferior FREQ_IN (Frequency inferior strata - FREQ_IN), Altura do estrato superior - ALT_SUP (Height of sward superior strata - HEI_SUP), Altura do estrato inferior - ALT_IN (Heigth of sward inferior strata - HEI_IN), Altura do estrato intermediário - ALT_INT(Height of sward intermediary strata-HEI_INT), Massa de forragem - MAS_FOR (Forage mass - MAS FOR), Freqüência E. Horridum - FREQ ER (Frequency E. Horridum - FREQ ER).

A análise de variância do tempo de pastejo não indicou diferença significativa $(\mathrm{P}<0,05)$ entre tratamentos (Tabela 2). Em princípio, nos tratamentos com baixa oferta de forragem, houve restrição de forragem, o que refletiu em aumento do tempo de pastejo pelo animal para compensar o consumo.

Nas ofertas de forragem maiores, quando consideradas todas as espécies da unidade experimental na avaliação de massa de forragem, possivelmente uma fração destas espécies não fez parte da dieta dos animais. Essa fração variou conforme cada unidade experimental, visto que sua estrutura e composição botânica é o resultado das intensidades de pastejo mantidas ao longo dos anos, de modo que a contribuição de espécies do estrato superior eleva com o aumento da oferta de forragem. Ribeiro Filho et al. (1997) observaram diminuição no tempo médio de pastejo de $0,43 \mathrm{~h} / \mathrm{kg}$ de MS de lâminas ofertadas por $100 \mathrm{~kg}$ de $\mathrm{PV} / \mathrm{dia}$ em uma pastagem de capim-elefante anão cultivar Mott. Esses autores obtiveram valores de tempo de pastejo que variaram de 312 a 582 minutos. Prates et al. (1995), no entanto, avaliando novilhos em uma pastagem nativa melhorada, observaram TP diurno de 300 a 474 minutos. O tempo de pastejo diurno, somado ao noturno,variou de 459 a 624 minutos. Portanto, as magnitudes observadas para o tempo de pastejo (Tabela 2) indicaram valores elevados para pastejo diurno, denotando situações de baixa disponibilidade de pastagem à medida que o tempo de pastejo e a disponibilidade de forragem estiveram inversamente rela cionados (Carvalho, 1997).
Tabela 2 - Tempo de pastejo por bovinos em pastagem natural com diferentes ofertas de forragem (OF)

Table 2 - Grazing time of cattle submitted to different herbage allowance $(H A)$ in native pasture

\begin{tabular}{|c|c|c|c|c|c|c|}
\hline \multirow[b]{2}{*}{4,0} & \multicolumn{6}{|c|}{$\begin{array}{l}\text { OF pretendida }(\mathrm{kg} \text { de } \mathrm{MS} / 100 \mathrm{~kg} \text { de } \mathrm{PV} / \mathrm{dia}) \\
\text { Targeted HA }(\mathrm{kg} D M / 100 \mathrm{~kg} \text { live weight/day })\end{array}$} \\
\hline & 8,0 & 12,0 & 16,0 & $8 \mathrm{p} 12$ & $12 \mathrm{p} 8$ & $16 \mathrm{p} 12$ \\
\hline \multirow{4}{*}{4,72} & \multicolumn{6}{|c|}{$\begin{array}{l}\text { OF real }(\mathrm{kg} \text { de } \mathrm{MS} / 100 \mathrm{~kg} \text { de } \mathrm{PV} / \mathrm{dia}) \\
\text { Actual HA }(\mathrm{kg} \mathrm{DM} / 100 \mathrm{~kg} \text { live weight/day })\end{array}$} \\
\hline & 12,35 & 17,71 & 23,53 & 13,71 & 15,40 & 17,83 \\
\hline & \multicolumn{6}{|c|}{ Tempo de pastejo (minutos) } \\
\hline & \multicolumn{6}{|c|}{ Grazing time (minutes) } \\
\hline $564^{\mathrm{a}}$ & $498^{\mathrm{a}}$ & $572^{\mathrm{a}}$ & $453^{\mathrm{a}}$ & $513^{\mathrm{a}}$ & $559^{\mathrm{a}}$ & $542^{\mathrm{a}}$ \\
\hline
\end{tabular}

Média seguidas de mesmas letras não diferem significativamente pelo teste Tukey $(\mathrm{P}>0,05)$.

( ) OF Real na ocasião das observações de pastejo.

Means followed by the same letters, in the same row, do not differ $(P>0.05)$ by Tukey test. () Actual HA during the grazing behaviour observation period.

Com a técnica multivariada, reduziu-se o número de fatores a serem analisados. Os eixos (fatores) são ilustrados em figuras, apresentadas em seqüência. Na Figura 1, as subfiguras (a) representam os vetores das variáveis e as subfiguras (b), as coordenadas dos tratamentos.

Os tratamentos de menor oferta de forragem $(4,0 \mathrm{e}$ $8,0 \%$ ) apresentaram maior freqüência de estrato inferior (Figura 1b). Maiores ofertas de forragem, fitomassas e freqüências de estrato superior foram observadas nos tratamentos de oferta de forragem média e alta, de 12,0 e 16,0\%, respectivamente. Na Figura 2, consta a relação linear positiva e negativa entre oferta de forragem, freqüência de estrato superior e frequiência de estrato inferior. 


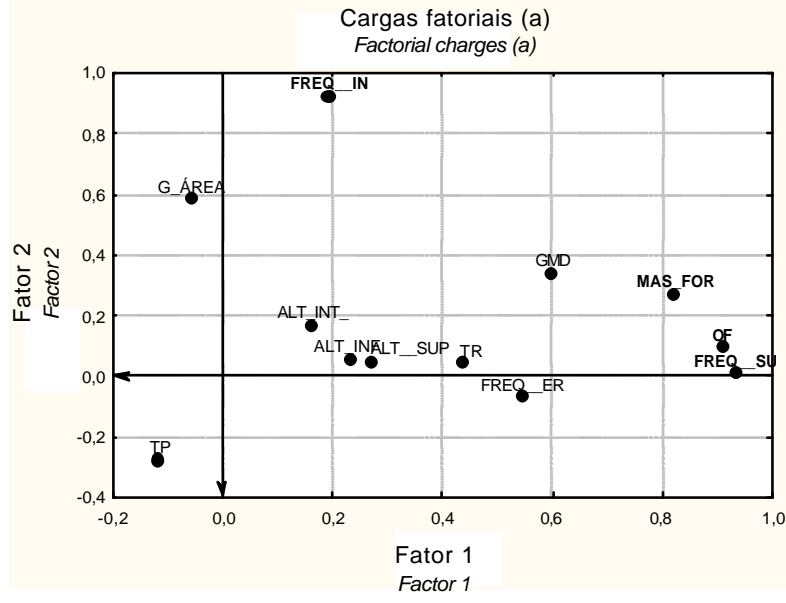

Figura 1 - Análise dos tratamentos: eixo 1 versus eixo 2.

Figure 1 - Treatment analysis: axis 1 versus axis 2.

Os resultados encontrados corroboram os descritos por Escosteguy (1990) e Moojen (2002), que verificaram que espécies como Paspalum notatum, $P$. paucifolium, $P$. pumilum aumentam a freqüência com a redução da $\mathrm{OF}$, enquanto A. lateralis, B. subaristata, C. selloana, B. trimera, A. filifolia, P. plicatulum, S. indicus, $D$. sericea e $D$. incanum aumentam a freqüência com altas OF.

Gonçalves e Girardi-Deiro (1988) descreveram aumento da freqüência de cobertura de Paspalum notatum de 26,9 para $62,9 \%$, à medida que aumentou a intensidade de pastejo.

O posicionamento da unidade experimental 8p12r2 na Figura $1 \mathrm{~b}$ indica um dossel composto por maior freqüência de estrato inferior, permanecendo mais próximo dos tratamentos com baixas ofertas que dos tratamentos com média e alta oferta de forragem.

O fator 3 (Figura 3a) indica relação inversa entre o tempo de pastejo e a altura do estrato inferior. O tempo de pastejo diminuiu à medida que a altura do estrato inferior aumentou. Os tratamentos $8 \mathrm{r} 2,8 \mathrm{p} 12 \mathrm{r} 1,12 \mathrm{p} 8 \mathrm{r} 2,16 \mathrm{p} 12 \mathrm{r} 1,16 \mathrm{r} 1$ e 16r2 proporcionaram ganho médio diário maior em tempos de pastejo menores (Figura 3b).

Esses tratamentos apresentaram ganho médio diário superior a $400 \mathrm{~g}$ e tempo de pastejo menor que 518 minutos (Tabela 1). Seguindo a indicação da análise multivariada, gerou-se uma equação de regressão com os tratamentos que não sofreram alteração da OFMST. A cada $1,0 \mathrm{~cm}$ de aumento do estrato inferior, houve diminuição de 66,7 minutos no tempo de pastejo, o que justifica sua importância para a composição da dieta dos animais em condições heterogêneas de pastejo (Figura 4). Minson (1983) verificou mecanismos integrados atuando no complexo planta-
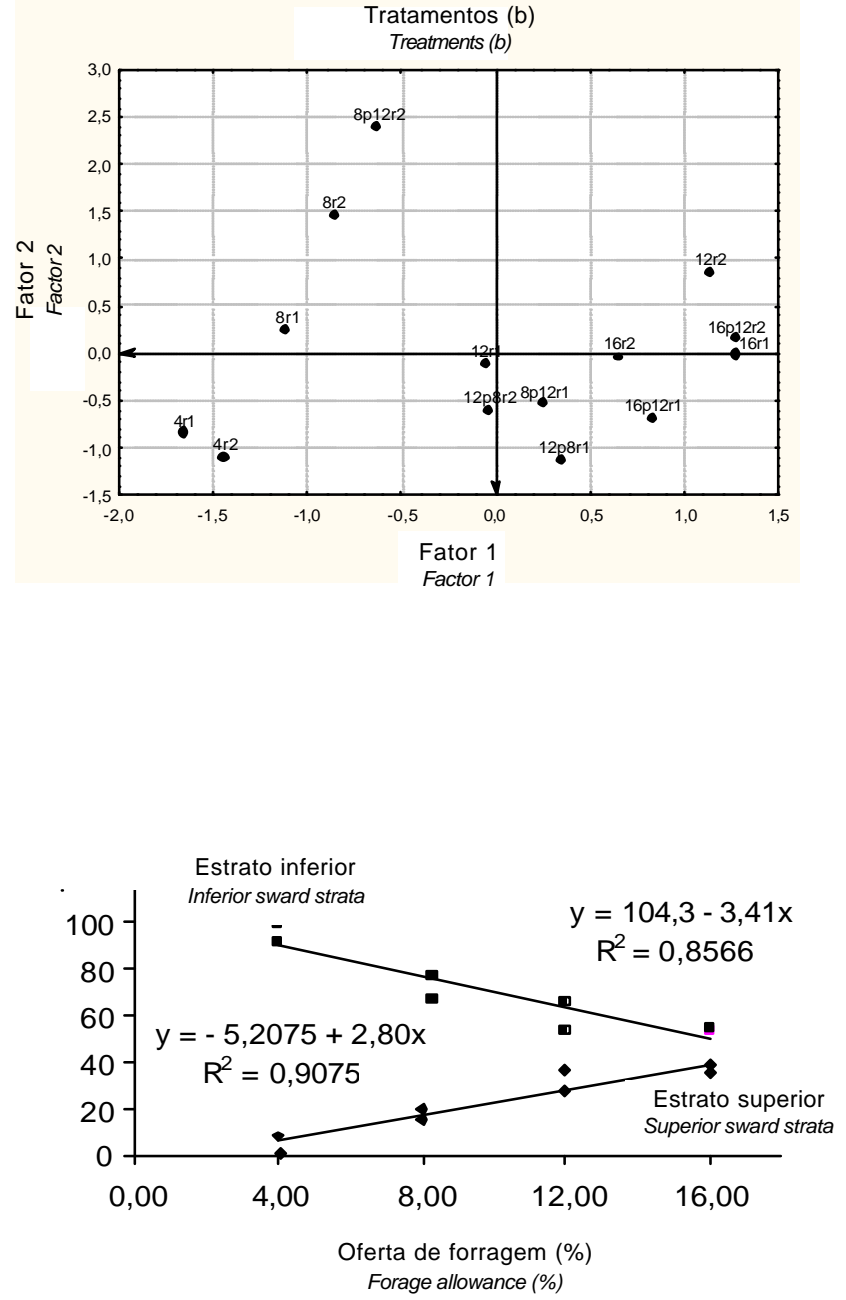

Figura 2 - Relação oferta de forragem versus freqüência dos estratos inferior e superior de uma pastagem nativa com diferentes ofertas de fitomassa aérea total $(\mathrm{kg}$ de $\mathrm{MS} / 100 \mathrm{~kg}$ de $\mathrm{PV} / \mathrm{dia})$.

Figure 2 - Relationship between herbage allowance and the frequency of superior and inferior sward strata from a native pasture submitted to different DM allowance $(\mathrm{kg} D M / 100 \mathrm{~kg}$ body weight/day).

animal, de modo que a qualidade da pastagem, assim como a disponibilidade do material preferido e sua acessibilidade, afetaram o consumo e, em cada condição da pastagem, uns ou outros assumiram o controle prioritariamente, provavelmente porque pode ocorrer mesma massa de forragem com inúmeras combinações de altura, densidade e composição (Carvalho et al. 1999).

Silveira (2001), avaliando alturas de pastejo com ovinos em azevém anual, e Castro (2003), em pastagem de milheto, encontraram resultados semelhantes, em que o tempo de pastejo variou linearmente à medida que elevou a altura da pastagem. Estes resultados corroboram as observações de Penning (1986), em pastagem de azevém perene cujas alturas tiveram importante influência sobre o comportamento dos animais em pastejo. 


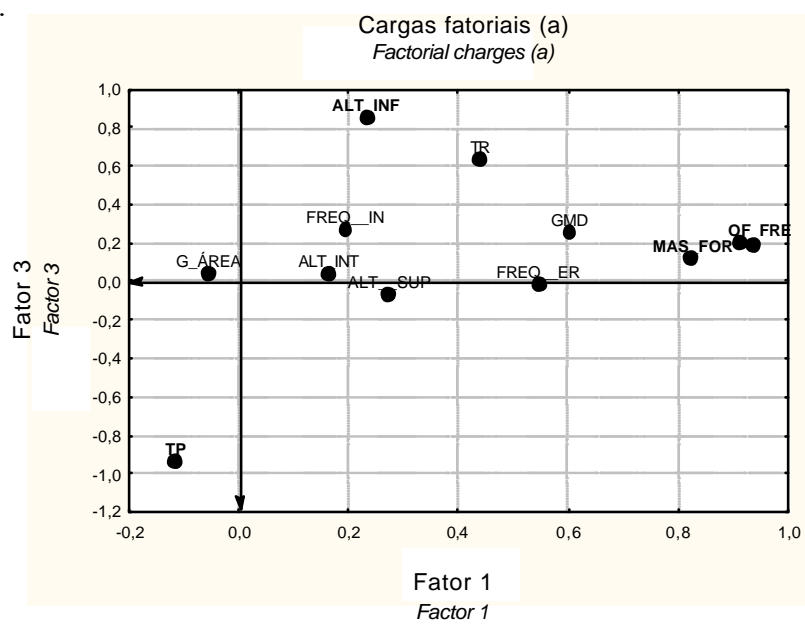

Figura 3 - Análise dos tratamentos: eixo 1 versus eixo 3 Figure 3 - Treatment analysis: axis 1 versus axis 3.

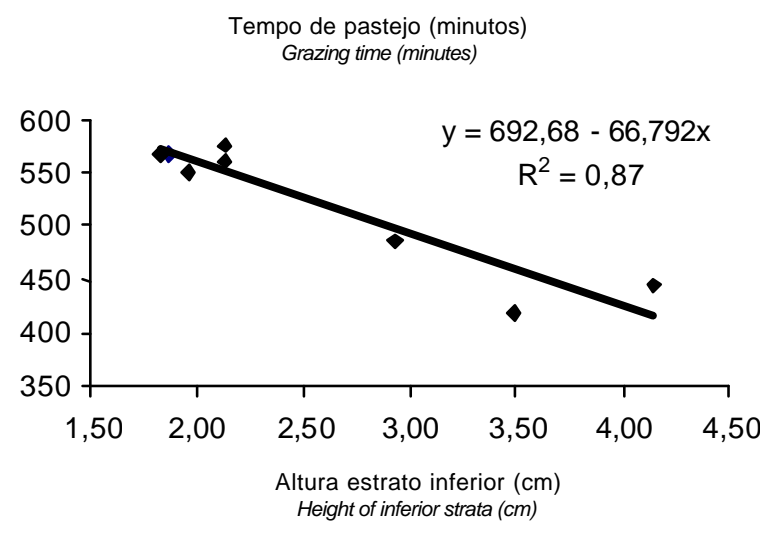

Figura 4 - Tempo de pastejo (minutos) de bovinos em uma pastagem nativa com diferentes ofertas de fitomassa aérea total ( $\mathrm{kg}$ de MS/ $100 \mathrm{~kg}$ de PV/dia).

Figure 4 - Grazing time (minutes) of cattle spent in the inferior stratum height $(\mathrm{cm})$ of native pasture submitted to different total DM allowance ( $\mathrm{kg} D M / 100 \mathrm{~kg}$ body weight/day).

Nota-se na Figura 5a a forma distinta com que cada repetição foi representada nos gráficos, comprovando a heterogeneidade entre as unidades experimentais quando comparados o fator 1 (massa de forragem, frequiência de estrato inferior e oferta de forragem) ao fator 4 (altura do estrato inferior).

O tempo de pastejo foi maior nos tratamentos com menor oferta de forragem, principalmente pela limitação de forragem imposta nestes tratamentos (Figura 6b).

O aumento do tempo de pastejoé uma tática dos animais para compensar situações de baixa disponibilidade de forragem. Neste estudo, a profundidade do bocado esteve provavelmente restringida, promovendo diminuição da

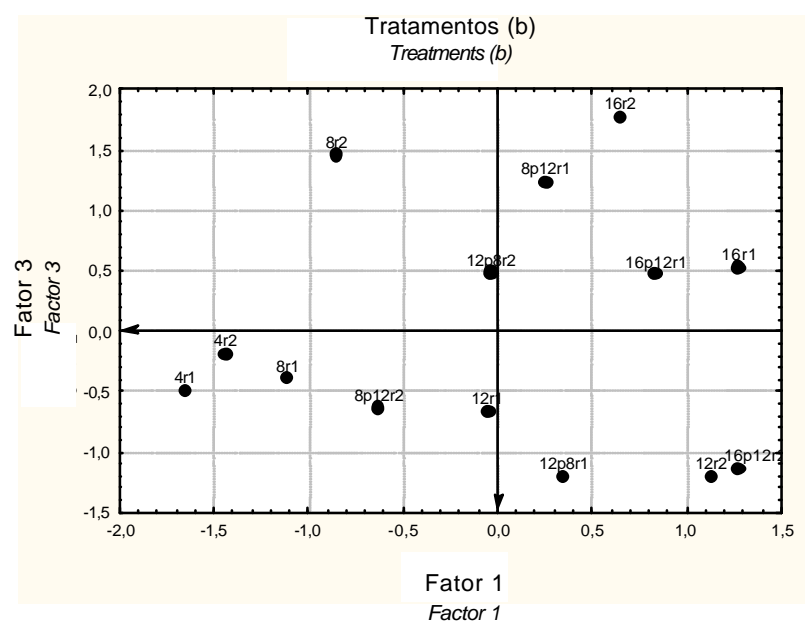

massa do bocado e, conseqüentemente, do consumo (Laca et al., 1992; Armstrong et al., 1995).

Os tratamentos com baixa oferta de forragem apresentaram freqüências de estrato inferior de 66,67 a $99.00 \%$; os tratamentos com alterações de oferta de forragem da prima vera para o verão apresentaram freqüência de estrato inferior que variou de 56,85 a $70,63 \%$, mais próximas das obtidas nos tratamentos de oferta de forragem fixa ao longo do ano (Figura 6b). O período de pastejo, com a alteração da oferta de forragem, não foi suficiente para induzir significativas mudanças no dossel das unidades experimentais moldadas ao longo dos anos. As unidades experimentais que apresentaram altura do estrato inferior menor que $2,42 \mathrm{~cm}$ apresentaram tempo de pastejo superior a 527,8 minutos.

Maior altura do estrato superior (figura $7 \mathrm{~b}$ ) foi observada nos tratamentos 12,0 e $16,0 \%$, nos quais a oferta de forragem não variou ao longo da estação de crescimento.

Verificou-se redução na altura do estrato superior quando a oferta de forragem foi alterada, comprovando que os animais foram forçados a consumir touceiras em maior grau, que, na condição anterior, com alta oferta de forragem, eram preteridas. Isso demonstra que é possível manipular a estrutura da pastagem por meio do ajuste da oferta de forragem.

Na Figura 8a, nota-se que o tempo de pastejo manteve-se em posição antagônica à altura do estrato inferior e à altura do estrato superior. Observando os dados da Tabela 1, verifica-se que, nas unidades experimentais com maior altura do estrato inferior, o tempo de pastejo foi menor. Observando a Figura 8b, nota-se que as unidades experimentais $8 \mathrm{r} 2,8 \mathrm{p} 12 \mathrm{r} 1$ e $16 \mathrm{p} 12 \mathrm{r} 1$ apresentaram maior altura do estrato inferior. 

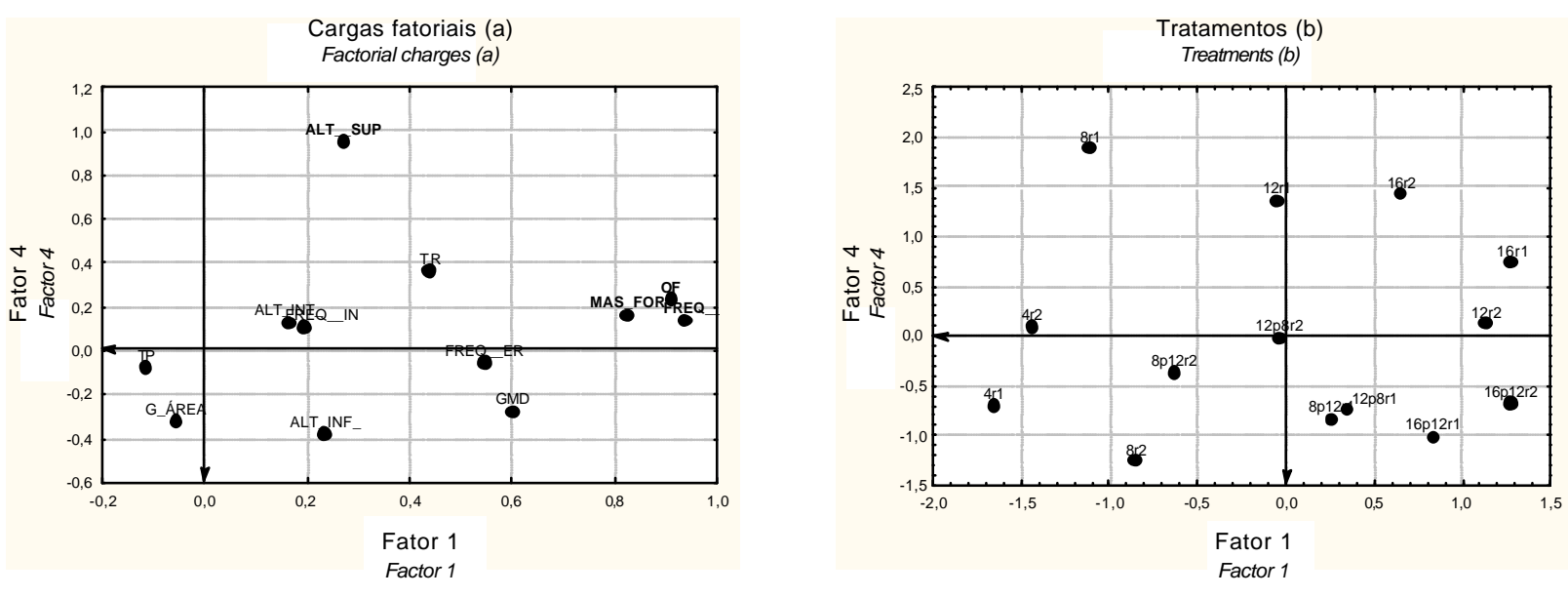

Figura 5 - Análise dos tratamentos: eixo 1 versus eixo 4.

Figure 5 - Treatment analysis: axis 1 versus axis 4.
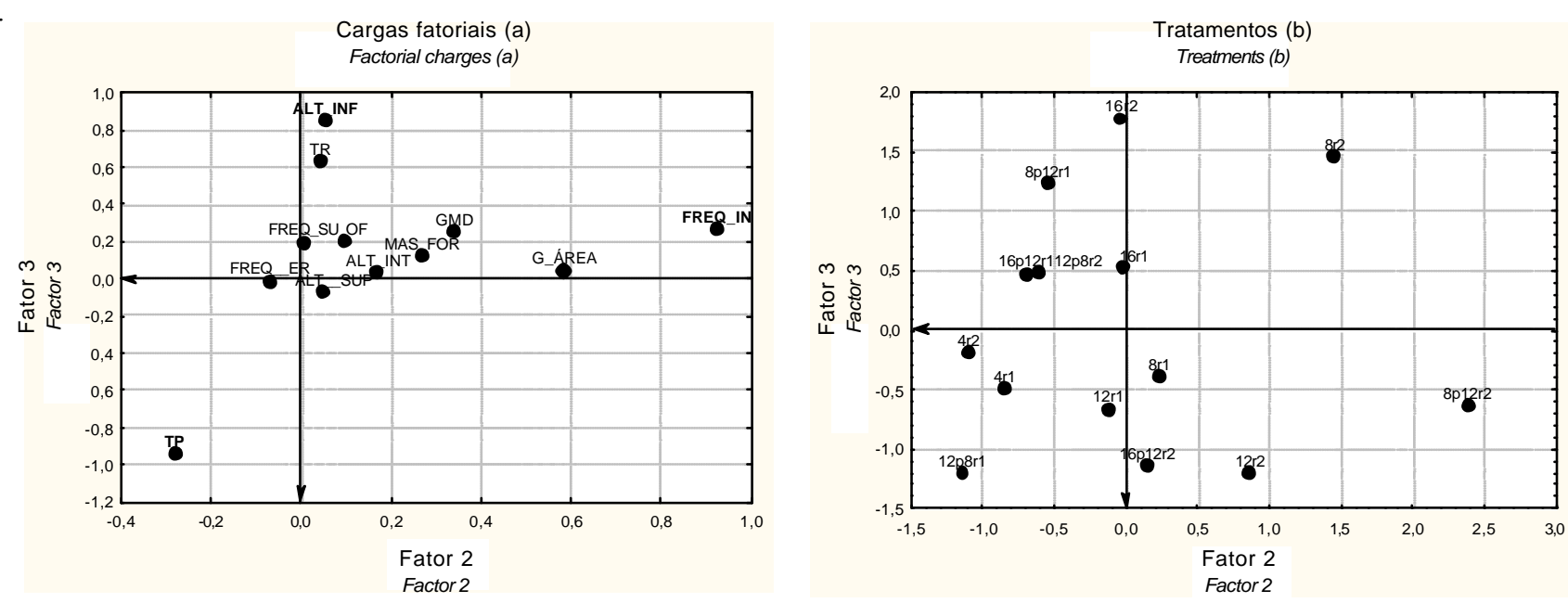

Figura 6 - Análise dos tratamentos: eixo 2 versus eixo 3.

Figure 6 - Treatment analysis: axis 2 versus axis 3.
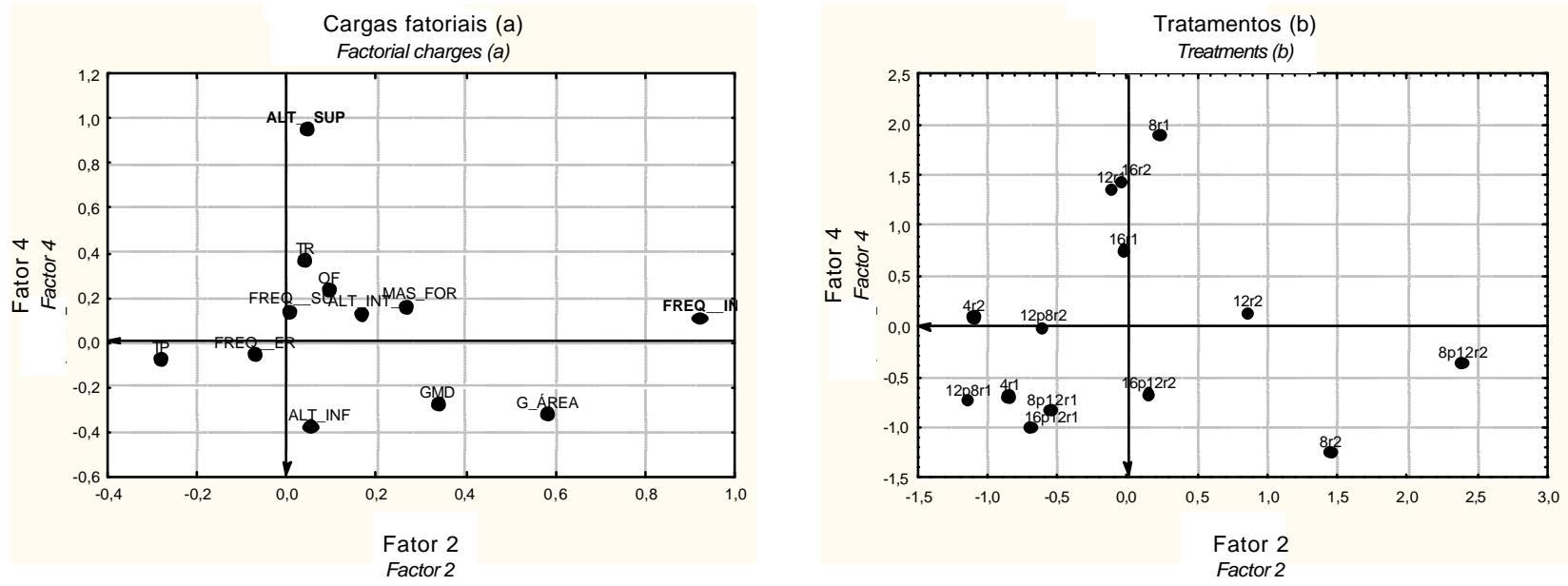

Figura 7 - Análise dos tratamentos: eixo 2 versus eixo 4.

Figure 7 - Treatment analysis: axis 2 versus axis 4 . 


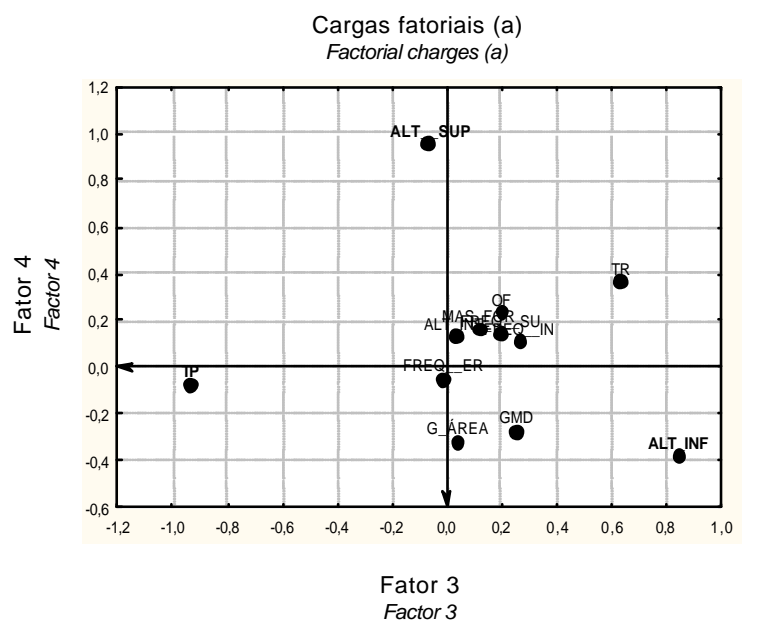

Figura 8 - Análise dos tratamentos: eixo 3 versus eixo 4. Figure 8 - Treatment analysis: axis 3 versus axis 4.

A oferta de forragem real manteve-se acima das pretendidas. Esta diferença foi maior à medida que aumentou a oferta de forragem do tratamento, principalmente em virtude da heterogeneidade das unidades experimentais, com es trutura e composição botânica distintas. Segundo Setelich (1994), a formação de touceira acarreta problemas metodológicos na avaliação de massa de forragem e no cálculo da OF. Este fato também é evidenciado pela distância com que as unidades experimentais desses tratamentos foram representadas em cada gráfico.

Metodologias que considerem a diversidade e complexidade das pastagens naturais devem ser utilizadas para correta avaliação e determinação do potencial produtivo deste ecossistema.

Com a utilização do método de análise dos componentes principais, encontraram-se importantes indicações sobre o comportamento ingestivo de bovinos em ambientes heterogêneos. Em consonância com o observado por Gordon (2000), em vegetação nativa da Escócia, o padrão de pastejo dos animais está intimamente relacionado à dinâmica dos estratos inferior e superior da pastagem, cujo estado e frequiência dependem das intensidades de pastejo empregadas. Estas evidências devem ser confirmadas e estudadas em pesquisas mais específicas para determinação dos fatores bióticos e abióticos que influenciam o comportamento em pastejo dos animais e da relevância desses fatores em vegetações complexas como as pastagens nativas.

\section{Conclusões}

Diferentemente do que ocorre em pastagens monoespecíficas, a oferta de forragem e a massa de forragem

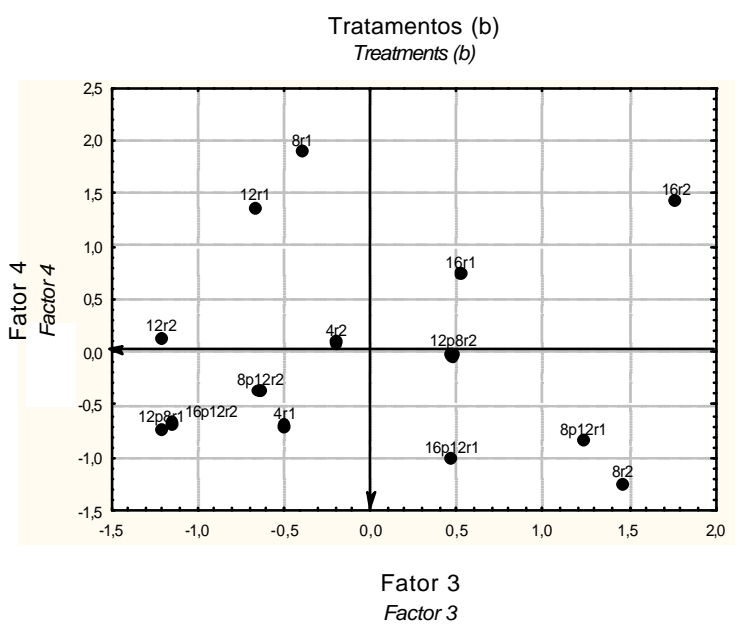

em pastagens nativas não são suficientes para explicar o tempo de pastejo dos animais.

Em ambientes de pastejo heterogêneos, com a presença de uma estrutura dupla bem definida, formada por um estrato inferior e outro superior, o tempo de pastejo e o ganho médio diário dependem mais da altura do estrato inferior que da oferta de forragem e da massa de forragem. Quanto menor a altura do estrato inferior, mais tempo é necessário para a seleção da dieta pelos animais.

A manipulação da estrutura por meio da alteração da oferta de forragem diminui a altura do estrato superior, indicando que a ocorrência de pastejo neste estrato pode ser manipulada pela variação da oferta de MS ao longo das estações do ano.

\section{Literatura Citada}

BOLDRINI, I.I. Campos do Rio Grande do Sul: caracterização fisionômica e problemática ocupacional. Porto Alegre: Instituto de Biociência/UFRGS, 1997. 39p. (Boletim, 56).

CORRÊA, F. Produção e qualidade de uma pastagem nativa do Rio Grande do Sul sob níveis de oferta de forragem a novilhos. Porto Alegre: Universidade do Rio Grande do Sul, 1993. 167p. Dissertação (Mestrado em Zootecnia) Universidade Federal do Rio Grande do Sul, 1993.

CARVALHO, P.C.F. A estrutura da pastagem e o comportamento ingestivo de ruminantes em pastejo. In: SIMPÓSIO SOBRE AVALIAÇÃO DE PASTAGENS COM ANIMAIS, 1., 1997, Maringá. Anais... Maringá: 1997. p.25-52.

ESCOSTEGUY, C.M.D. Avaliação agronômica de uma pastagem natural sob níveis de pressão de pastejo. Porto Alegre: Universidade do Rio Grande do Sul, 1990. 231p. Dissertação (Mestrado em Zootecnia) - Universidade Federal do Rio Grande do Sul, 1990.

GORDON, I.J. Plant-animal interactions in complex communities: from mechanism to modelling. In: HODGSON J.; LEMAIRE G.; MORAES, A. et al. (Eds.). Grassland ecophysiology and grazing ecology. Wallingford: CAB International, 2000. p.191-207. 
HAYDOCK, K.P.; SHAW, N.H. The comparative yield method for estimating dry matter yield of pasture. Australian Journal Agricultural and Animal Husbandry, v.15, p.66-70, 1975.

MARASCHIN, G.E. Manejo de pastagens nativas, produtividade animal e dinâmica da vegetação em pastagens nativas do Rio Grande do Sul. In: REUNIÃ̃O DO GRUPO TÉCNICO EM FORRAGEIRAS DO CONE SUL - ZONA CAMPOS, 17., 1998, Lages. Anais... Lages: Epagri/UDESC, 1998. p.47-54.

MINSON, D.J. Forage quality: assessing the plant-animal complex. In: INTERNACIONAL GRASSLAND CONGRESS, 14., 1996, Sydney. Proceedings... Sydney, 1996. p.23-29.

MOOJEN, E.L.; MARASCHIN, G.E. Potencial produtivo de uma pastagem nativa do Rio Grande do Sul submetida a níveis de oferta de forragem. Ciência Rural, v.32.n.1, p.127-132, 2002.

POPPI, D.P.; HUGUES, J.P.; L'HUILLIER, P.J. Intake of pasture by grazing ruminants. In: NICOL, A.M. (Ed.). Feeding livestock on pasture. New Zealand: Society of Animal Production, 1987. p.55-63.

PRATES, E.R.; BONELI, I.B.; PIAGGIO, L.M. et al. Tempo e ciclos de pastejo de novilhos mantidos em condições de pastagem nativa melhorada. Revista da Sociedade Brasileira de Zootecnia, v.24, n.1, p.1-7, 1995.

RIBEIRO FILHO, H.M.N.; ALMEIDA, E.X.; HARTHMANN, O.E.L. et al. Tempo e ciclos de pastejo de bovinos submetidos a diferentes ofertas de capim elefante anão cv. "Mott". In.: REUNIÃO ANUAL DA SOCIEDADE BRASILEIRA DE ZOOTECNIA, 34., 1997. Juiz de Fora. Anais... Juiz de Fora: Sociedade Brasileira de Zootecnia, 1997. p.276-278.
SETELICH, H.A. Potencial produtivo de uma pastagem natural do Rio Grande do Sul, submetida a distintas ofertas de forragem. Porto Alegre: Universidade Federal do Rio Grande do Sul, 1994. 169p. Dissertação (Mestrado em Zootecnia) - Universidade Federal do Rio Grande do Sul, 1994.

STATISTICAL ANALYSIS SYSTEM - SAS. System for Information: versão 6.11. Cary: 1996.

SOARES, A.B.; CARVALHO, P.C.F.; NABINGER, C. et al. Produção animal e de forragem em uma pastagem native submetida a distintas ofertas de forragem. Ciência Rural, v.35, p.1148$1154,2005$.

STATSOFT. STATISTICA for Windows (Computer program manual). Tulsa: StatSoft Inc., 1998. (CD-ROM).

WILM, H.G.; COSTELLO, D.F.; KLIPPE, G.E. Estimating forage yield by the double sampling method. Journal of the American Society of Agronomy, v.36, n.1, p.194-203, 1944. 